\title{
Correlation between NT-proBNP, hsCRP and OXSR1: Clinical relevance in evaluating the prognosis of polytrauma patients
}

\author{
Costin Dumitru',2, Alexandra Ripszky Totan², Iulia-Ioana Stanescu-Spinu², \\ Daniela Miricescu², Maria Greabu ${ }^{2}$ \\ ${ }^{1}$ Bucharest Emergency Clinical Hospital, Bucharest, Romania \\ 2Department of Biochemistry, Faculty of Dental Medicine, "Carol Davila" University of Medicine and \\ Pharmacy, Bucharest, Romania
}

\begin{abstract}
Introduction. Polytrauma patients are expected to have a higher risk of mortality than that obtained by the summation of expected mortality owing to their individual injuries. Subsequent life-threatening posttraumatic complications are associated with overproduction of proinflammatory mediators (e.g. cytokines, chemokines) and the critical imbalance of cell-regulated innate immunity.

Material and methods. The present study is aimed to identify a possible relationship between NT-proBNP, hs-CRP and OXSR1 biochemical markers in polytrauma patients, both by bibliographic research and quantitative analysis and statistics of some parameters of interest in lot of 46 patients.

Results and discussions. The correlation between hsCRP and the inflammatory response after trauma is well-known and well documented in literature, so the negative correlation between hsCRP and OXSR1 that resulted from our analysis is of great significance resulting in a potentially new biomarker to be further studied and used in determining the possible outcome of polytrauma patients.

Conclusion. Although OXSR1 is a new in the field of polytrauma patients, because is negatively correlated with hsCRP and the clinical evolution of the patients we think that OXSR1 is a good biomarker to further in investigate and may be used in determining the clinical progress of the polytrauma patients.
\end{abstract}

\section{Keywords: inflammation, polytrauma, hsCRP, NT-proBNP, OXSR1}

\section{INTRODUCTION}

Polytrauma patients are expected to have a higher risk of mortality than that obtained by the summation of expected mortality owing to their individual injuries [1]. The immunological response to severe injury and multiple trauma remains a serious challenge in critical care management. Subsequent life-threatening posttrau-matic complications are associated with overproduction of proinflammatory mediators (e.g. cytokines, chemokines) and the critical imbalance of cell-regulated innate im- munity. In prolonged deregulated immune cell homeostasis, the immunological sequelae commonly evolve a state of hyperinflammation or immunosuppression or both, ultimately leading to multiple organ dysfunction syndrome (MODS) or lethal failure (MOF -multiple organ failure) [2]. Although the hyperinflammatory response within the innate immune reaction to multiple trauma is not inevitable, it characteristically involves exaggerated release of proinflammatory mediators. These include interleukin (IL)-1, IL-6, IL-8 and IL-18, tumor necrosis 
factor (TNF)-a, neutrophil activation, microvascular adherence and an uncontrolled polymorphonuclear (PMN) and macrophage oxidative burst [3].

Some studies have found that serum atrial natriuretic peptide (ANP) and $\beta$-type natriuretic peptide (BNP) levels exhibit marked increases in numerous types of disease, including heart failure, acute lung injury (ALI), septic shock, burns and electrical injury, and that these increases are closely associated with the hemodynamic index [4,5]. Compared with traditional inflammatory markers and trauma scoring systems, however, the correlation between the levels of serum $\mathrm{N}$-terminal peptide of pro-ANP (NT-proANP) and N-terminal fragment of BNP (NT-proBNP), which are used as indicators of the trauma-induced development of MODS, and the hemodynamic index following severe trauma is rarely reported. The aim of the present study, therefore, was to evaluate the prognostic value of serum NT-BNP in severe trauma patients with or without MODS and to perform a correlation analysis between these indicator and the clinical evolution of the patients, hs-CRP and OXSR-1 [6].

Several studies have shown that NT-proBNP could serve as an independent predictor of greater mortality in patients with cardiogenic shock [7], septic shock [8], severe sepsis [9], as well as in noncardiac [10] or unselected patients [11], while another study [12] demonstrated that NT-proBNP failed to predict short-term mortality of ICU patients with hypoxic respiratory failure [13].

CRP is a positive, acute-phase protein produced by hepatocytes in response to inflammatory conditions. CRP levels quickly increase in response to trauma, inflammation, and infection and decrease just as quickly as the body condition improves. Therefore, CRP levels are widely used to monitor various inflammatory conditions [14,15].

Oxidative stress-responsive kinase 1 (OXSR1) belongs to the Serine/Threonine protein kinase family and plays a crucial role in regulating diverse cellular pathophysiologic functions, including ion homeostasis, development, differentiation, angiogenesis, invasive migration, and metastasis [16].

\section{MATERIAL AND METHODS}

The present study is part of the research carried out during the doctoral internship, performed on a group of patients, in which we aimed to identify a possible relationship between NT-proBNP, hs-CRP and OXSR1 biochemical markers in polytrauma patients, both by bibliographic research and quantitative analysis and statistics of some parameters of interest. All the participants, patients and controls, provided the informed consent to participate in the study and we obtained the approval of the Ethics
Committee of the Bucharest Emergency Clinical Hospital no 6886/12.07.2021.

Criteria for inclusion in the study: patients who have suffered a trauma due to a high-energy traumatic agent that had an Injury Severity Score (ISS) > 16 and associated a pelvic ring fracture. And healthy patients, with no traumatic event in the last 4 weeks and no recent history of inflammatory disease or infection.

Exclusion criteria from the study: the presence of acute infectious diseases; the existence of a chronic infectious disease; patients who have suffered a heart attack or stroke in the last 4 weeks; active cancers with damage to organs or soft tissues; the presence of an autoimmune inflammatory disease.

Blood samples were collected in usual matter approximatively $5 \mathrm{ml}$ of blood in red-cap Vacutainer for serum samples preparation for all analyzed parameters (NT-pro-BNP, hsCRP and OXSR1) were performed from a lot of 46 patients - 24 patients who have suffered a trauma due to a high-energy traumatic agent; patients with ISS $>16$ that associated a pelvic ring fracture, and 22 patients control lot of healthy patients that meet the inclusion criteria for this group. The average age of the patients in the two groups was: $35+/-7$ years for the patients who suffered a high energy trauma, and $37+/-11$ years for the patients in the control group.

For determining the serum levels of OXSR1 and NT-pro-BNP we have used ELISA assay kits from Cusabio (Wuhan Hi-tech Medical Devices Park China) and a semiautomatic immunoassay system STAT FAX 303 PLUS (Awareness Technologies - USA). Serum hsCRP levels have been measured on a chemiluminescence automatic analyzer, Maccura1200 Plus (China).

Statistical analysis was performed using IBM SPSS Statistics 25 and Microsoft Office Excel / Word 2013. Quantitative variables were tested for distribution using the Shapiro-Wilk test. The correlations established between them were performed using the Spearman's rho correlation coefficient, relative to the non-parametric distribution of the variables.

\section{RESULTS}

The results of the three studied parameters and the correlations between them are illustrated in tables 1 to 3 and Figures 1 to 3 .

TABLE 1. Correlation between NT-proBNP values and hsCRP values

\begin{tabular}{|c|c|}
\hline Correlation & $\mathrm{p}^{*}$ \\
\hline NT-proBNP $\left(\mathrm{p}=0.159^{* *}\right) \times$ hsCRP & 0.419, \\
$\left(\mathrm{p}=0.004^{* *}\right)$ & $\mathrm{R}=0.145$ \\
\hline
\end{tabular}


The data in Table 1 and Figure 1 represent the correlation between NT-proBNP values and hs CRP values. The hs CRP values have a non-parametric distribution according to the Shapiro-Wilk test $(\mathrm{p}=$ $0.004)$. The observed correlation is insignificant $(p=$ $0.419, \mathrm{R}=0.145$ ), so that in the studied group, there was no significant association between NT-proBNP values and hs CRP values.

TABLE 2. Correlation between NT-proBNP values and OXSR1 values

\begin{tabular}{|c|c|}
\hline Correlation & $\mathbf{p}^{*}$ \\
\hline NT-proBNP $\left(p=0.159^{* *}\right) \times$ OXSR1 & 0.770 \\
$\left(p=0.030^{* *}\right)$ & $\mathrm{R}=0.053$ \\
\hline
\end{tabular}

*Spearman's rho correlation coefficient, **Shapiro-Wilk test

The data in Table 2 and Figure 2 represent the correlation between NT-proBNP values and OXSR1 values. OXSR1 values have a non-parametric distribution according to the Shapiro-Wilk test $(\mathrm{p}=0.030)$. The observed correlation is insignificant $(p=0.770$, $\mathrm{R}=0.053$ ), so that in the studied group, there was no significant association between NT-proBNP values and OXSR1 values.

TABLE 3. Correlation between hs CRP values and OXSR1 values

\begin{tabular}{|c|c|}
\hline Correlation & $\mathbf{p}^{*}$ \\
\hline $\operatorname{hsCRP}\left(p=0.004^{* *}\right) \times$ OXSR1 $\left(p=0.030^{* *}\right)$ & 0.002, \\
$R=-0.517$ \\
\hline
\end{tabular}

*Spearman's rho correlation coefficient, **Shapiro-Wilk test

The data in Table 3 and Figure 3 represent the correlation between the hs CRP values and the
OXSR1 values. Both variables have a non-parametric distribution according to the Shapiro-Wilk test $(p<0.05)$. The observed correlation is significant and negative, of high degree $(p=0.002, R=-0.517)$, observing in the studied group, that patients with high hs CRP values are significantly more frequently associated with lower OXSR1 values and vice versa.

\section{DISCUSSIONS}

Polytrauma is generally used to describe trauma patients whose injuries involve multiple body regions, compromise the patient's physiology and potentially cause dysfunction of uninjured organs [17]. The expected higher risk of mortality of polytrauma patients is based on the assumption that the underlying pathophysiological response of the injured person would aggravate the clinical outcome. The injured person's pathophysiological response to the injury load, however, makes a differentiation between "polytrauma" and "multitrauma" [18]. For example, many reports had indicated that head and brain injuries and thoracic traumata are major risk factors in trauma patients and that co-occurrence of these factors leads to an exponential increase in mortality [1].

The hsCRP has been noted to have opsonizing properties, increasing the recruitment of monocytes and also inducing endothelial dysfunction by suppressing basal and induced nitric oxide release and therefore is an excellent indicator of systemic inflammation. The hsCRP per se has also been found to increase the expression of vascular endothelial plasminogen activator inhibitor-1 (PAI-1) [19].

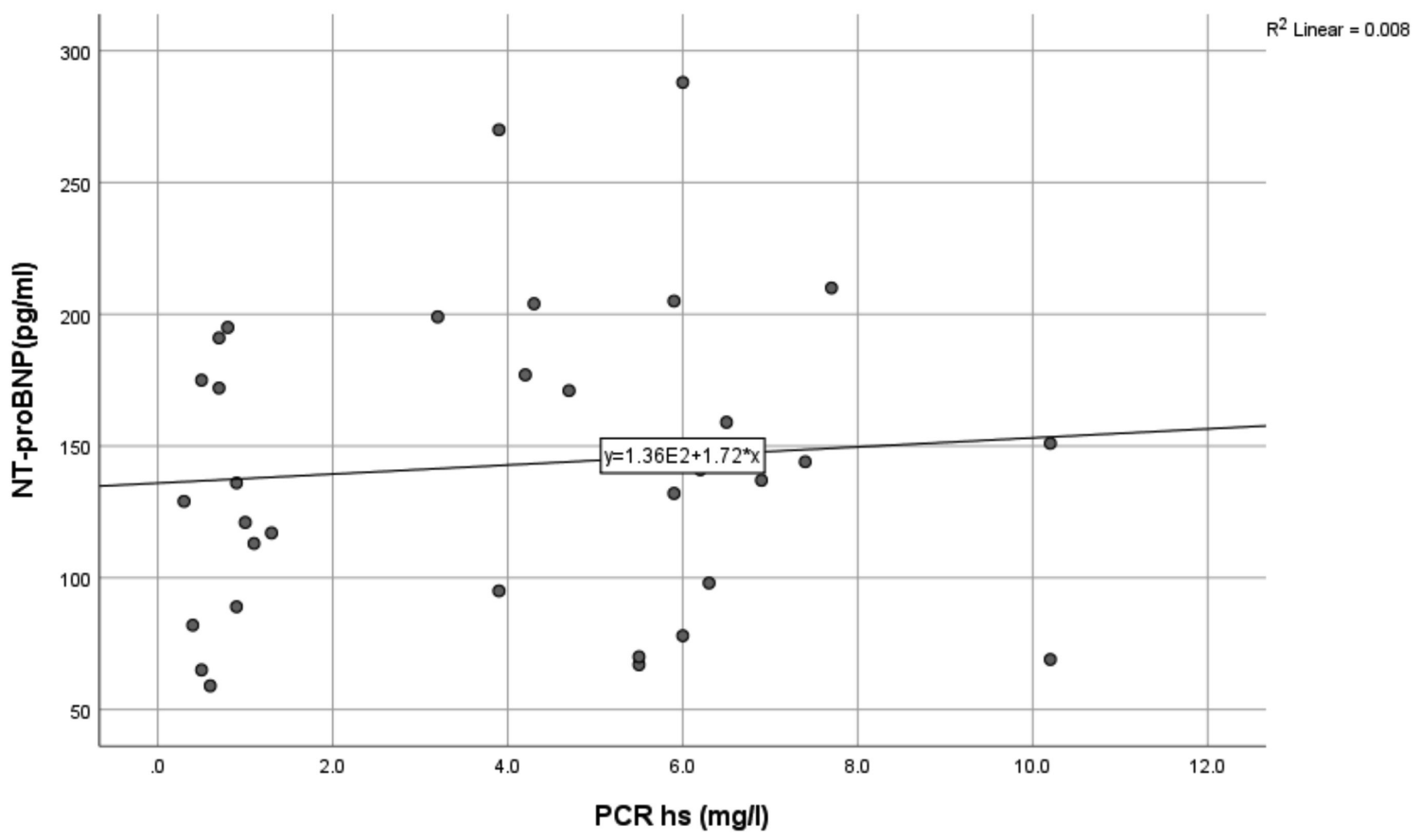

FIGURE 1. Correlation between NT-proBNP values and hs CRP values 


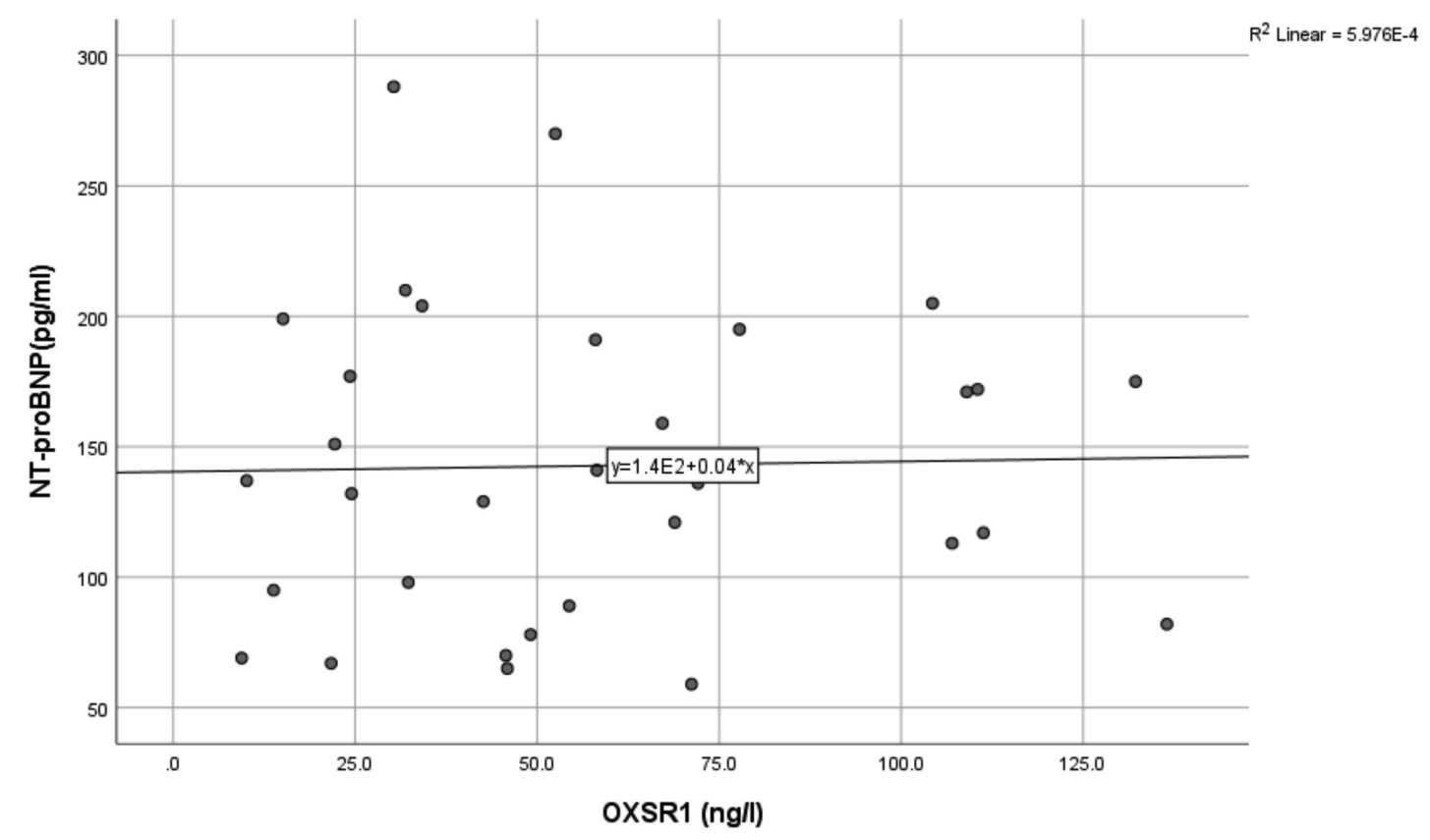

FIGURE 2. Correlation between NT-proBNP values and OXSR1 values

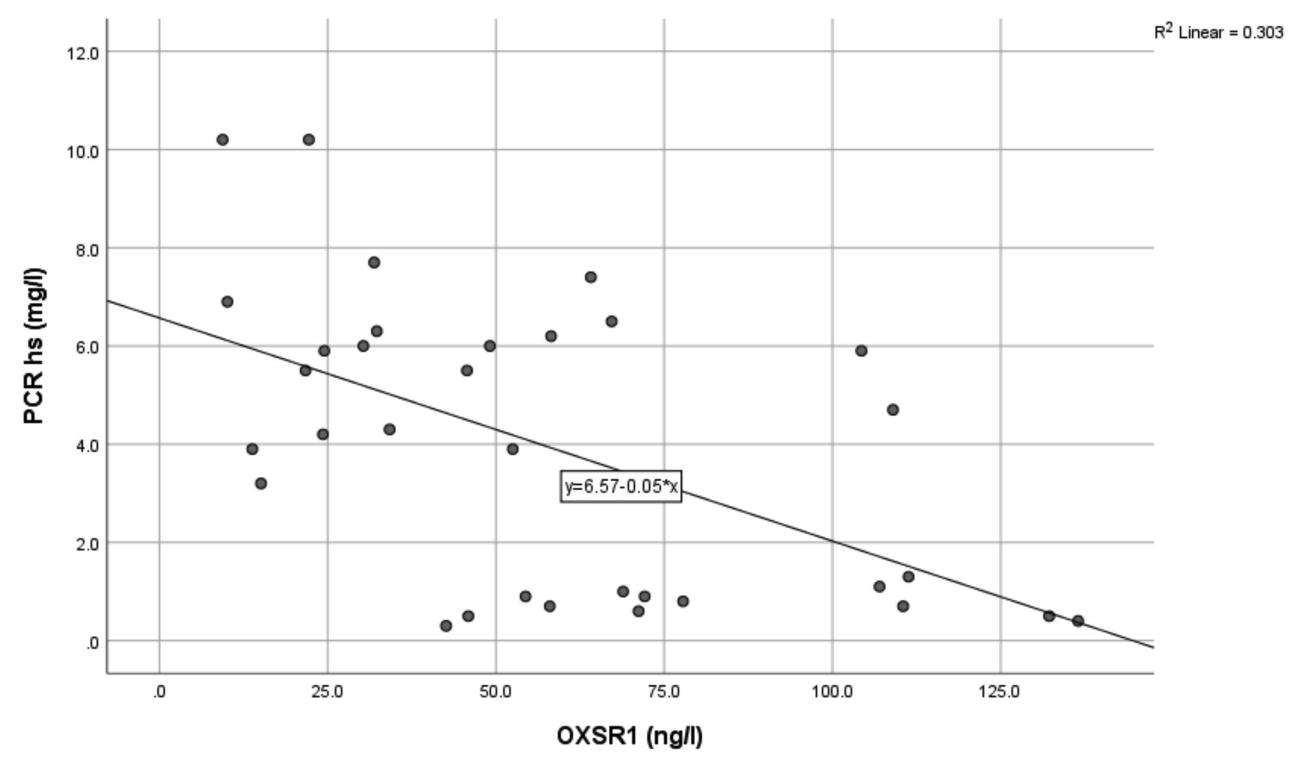

FIGURE 3. Correlation between hs CRP values and OXSR1 values

NT-proBNP and CRP caused by excessive inflammatory reactions in trauma patients may affect their efficiency for prediction outcome and could be easily influenced by many factors, such as certain medications (e.g., steroids that are frequently used in these cases). The correlation between hsCRP and the inflammatory response after trauma is well-known and well documented in literature, so the negative correlation between hsCRP and OXSR1 that resulted from our analysis is of great signifi- cance resulting in a potentially new biomarker to be further studied and used in determining the possible outcome of polytrauma patients.

Despite the fact that our study enrolled a small number of patients and is only at the beginning, the results obtained seem very promising and encourage us to continue the study, and we consider ourself pioneers in evaluating this correlation of biomarkers in polytrauma patients. 


\section{CONCLUSION}

Although OXSR1 is a new in the field of polytrauma patients, the fact that is negatively correlated with hsCRP and the clinical evolution of the patients we think that is a good biomarker to further in investigate and maybe use in determining the clinical progress of the polytrauma patient.

\section{Conflict of interest: none declared Financial support: none declared}

\section{REFERENCES}

1. Rau CS, Wu SC, Kuo PJ, Chen YC, Chien PC, Hsieh HY, Hsieh CH. Polytrauma Defined by the New Berlin Definition: A Validation Test Based on Propensity-Score Matching Approach. Int J Environ Res Public Health. 2017 Sep 11;14(9):1045.

2. Keel M, Trentz O. Pathophysiology of polytrauma. Injury. 2005 Jun;36(6):691-709.

3. Tschoeke SK, Ertel W. Immunoparalysis after multiple trauma. Injury. 2007 Dec;38(12):1346-57.

4. Determann RM, Royakkers AA, Schaefers J, de Boer AM, Binnekade JM, van Straalen JP, Schultz MJ. Serum levels of N-terminal proB-type natriuretic peptide in mechanically ventilated critically ill patients-relation to tidal volume size and development of acute respiratory distress syndrome. BMC Pulm Med. 2013 Jul 9;13:42.

5. Papanikolaou J, Makris D, Mpaka M, Palli E, Zygoulis P, Zakynthinos E. New insights into the mechanisms involved in B-type natriuretic peptide elevation and its prognostic value in septic patients. Crit Care. 2014 May 9;18(3):R94.

6. Li N, Song Z, Wang J, Teng Y, Cui Y, Jin H, Gao Y. Prognostic value of natriuretic peptides in severe trauma patients with multiple organ dysfunction syndrome. Exp Ther Med. 2015 Aug;10(2):792-796.

7. Januzzi JL, Morss A, Tung R, Pino R, Fifer MA, Thompson BT, LeeLewandrowski E. Natriuretic peptide testing for the evaluation of critically ill patients with shock in the intensive care unit: a prospective cohort study. Crit Care. 2006 Feb;10(1):R37.

8. Roch A, Allardet-Servent J, Michelet P, Oddoze C, Forel JM, Barrau K, Loundou A, Perrin G, Auffray JP, Portugal H, Papazian L. NH2 terminal pro-brain natriuretic peptide plasma level as an early marker of prognosis and cardiac dysfunction in septic shock patients. Crit Care Med. 2005 May;33(5):1001-7.

9. Varpula M, Pulkki K, Karlsson S, Ruokonen E, Pettilä V; FINNSEPSIS Study Group. Predictive value of N-terminal pro-brain natriuretic peptide in severe sepsis and septic shock. Crit Care Med. 2007 May;35(5):1277-83.

10. Shah KB, Nolan MM, Rao K, Wang DJ, Christenson RH, Shanholtz CB, Mehra MR, Gottlieb SS. The characteristics and prognostic importance of NT-ProBNP concentrations in critically ill patients. Am J Med. 2007 Dec;120(12):1071-7.

11. Meyer B, Huelsmann M, Wexberg P, Delle Karth G, Berger R, Moertl D, Szekeres T, Pacher R, Heinz G. N-terminal pro-B-type natriuretic peptide is an independent predictor of outcome in an unselected cohort of critically ill patients. Crit Care Med. 2007 Oct;35(10):2268-73.

12. Jefic $D$, Lee JW, Jefic D, Savoy-Moore RT, Rosman HS. Utility of B-type natriuretic peptide and $\mathrm{N}$-terminal pro $\mathrm{B}$-type natriuretic peptide in evaluation of respiratory failure in critically ill patients. Chest. 2005 Jul;128(1):288-95.

13. Wang F, Pan W, Pan S, Wang S, Ge Q, Ge J. Usefulness of N-terminal pro-brain natriuretic peptide and C-reactive protein to predict ICU mortality in unselected medical ICU patients: a prospective, observational study. Crit Care. 2011;15(1):R42.

14. Marnell L, Mold C, Du Clos TW. C-reactive protein: ligands, receptors and role in inflammation. Clin Immunol. 2005 Nov;117(2):104-11.

15. Du Clos TW, Mold C. C-reactive protein: an activator of innate immunity and a modulator of adaptive immunity. Immunol Res. 2004; 30(3):261-77.

16. Li Y, Qin J, Wu J, Dai X, Xu J. High expression of OSR1 as a predictive biomarker for poor prognosis and lymph node metastasis in breast cancer. Breast Cancer Res Treat. 2020 Jul;182(1):35-46.

17. Pape HC, Lefering R, Butcher N, Peitzman A, Leenen L, Marzi I, Lichte $P$, Josten C, Bouillon B, Schmucker U, Stahel P, Giannoudis P, Balogh Z. The definition of polytrauma revisited: An international consensus process and proposal of the new 'Berlin definition'. J Trauma Acute Care Surg. 2014 Nov;77(5):780-786.

18. Butcher N, Balogh ZJ. The definition of polytrauma: the need for international consensus. Injury. 2009 Nov;40 Suppl 4:S12-22.

19. Kamath DY, Xavier D, Sigamani A, Pais P. High sensitivity C-reactive protein (hsCRP) \& cardiovascular disease: An Indian perspective. Indian J Med Res. 2015 Sep;142(3):261-8. 